\title{
TEMATOLOGÍA Y TRANSTEXTUALIDAD
}

\section{Problemas de definición}

La tematología es una rama de la literatura comparada que estudia aquella dimensión abstracta de la literatura que son los materiales de que está hecha, así como sus transformaciones y actualizaciones; estudia, en otras palabras, los temas y motivos que, como filtros, seleccionan, orientan e informan el proceso de producción de los textos literarios.

Durante mucho tiempo se pensó que el estudio de temas era "extrínseco" a la literatura y provincia exclusiva de la etnoliteratura, pero una vez que fueron aceptadas las relaciones de afinidad y no sólo las de derivación e influencia, la literatura comparada hizo suyo el estudio de temas y motivos. Hoy en día, cuando aquella división tajante entre lo "intrínseco" y lo "extrínseco"' en los estudios literarios ya no nos parece tan nítida o pertinente consideramos con Harry Levin que "a writer's choice of a subject is a aesthetic decision"'2 que afecta de manera importante la significación del texto. Más aún, el tema no es sólo una elección por parte del escritor sino una construcción por parte del lector, como bien lo ha observado Claudio Guillén, pues es de hecho el lector o el crítico

quien sin cesar elige, extrae, cita, es decir... [que] las formas y los temas, más que entidades discretas, son elementos parciales cuyo montaje se debe en definitiva a la intervención del lector. Tratándose de tematología, esta intervención será tanto más

${ }^{1}$ Cf. Wellek \& Warren 1949.

${ }^{2}$ Levin 1968, p. 145. 
importante cuanto más... relevantes los fenómenos de intertextualidad que identifiquen el tema mediante la memoria de figuraciones anteriores ${ }^{3}$.

La tematología opera, así, una especie de reagrupación de los textos literarios desde una perspectiva temática.

No habremos de abordar aquí el conflicto terminológico, aún hoy no resuelto, entre las escuelas alemana y anglosajona. Lo que para los unos es tema para los otros es motivo y viceversa. Es del todo preferible encontrar las bases semióticas que permitan una conciliación y una mayor claridad conceptual. Comenzaremos por dar definiciones de uso corriente, antes de llegar al sentido más especializado que tienen estos términos en tematología.

Un tema se define como "asunto, materia, cosa de la que se trata en una conversación, escrito, conferencia, etc." "4. El terna tendría entonces un valor abstracto: la materia prima a desarrollar en un discurso. Motivo en cambio se define de diversas maneras: en música, contradictoriamente, se define como "Tema. Melodía principal, que se repite en distintas formas, en una composición. Por extensión, pensamiento que se repite o que está presente en todo el desarrollo de una obra de la mente" ; en decoración y escultura, el motivo es la "figura dibujada o esculpida que constituye un detalle, en general repetido, de la decoración" 5 . También se ha definido el motivo como el rasgo constitutivo de una composición; como el objeto o conjunto de objetos que configuran un elemento distintivo del diseño. Finalmente, el motivo sería definido como un principio estructural, como la idea dominante de una obra. En una composición literaria el motivo se define, no como la causa, o "motor", de una acción; desde la perspectiva de su composición, un motivo constituye, más bien, un incidente, una situación particular, un problema ético ${ }^{6}$. Ya incluso en estas definiciones de uso corriente, la relación entre tema y motivo no es una de oposición entre lo abstracto y lo concreto, puesto que tanto el tema como el motivo tienen un carácter abstracto y de materia prima. Lo que los diferencia en un primer momento es que el tema, en tanto que asunto o materia del discurso, orienta una posible selección de incidentes o detalles que permita su desarrollo; el motivo en cambio se distingue

${ }^{3}$ Guillén 1985, p. 249.

${ }^{4}$ Moliner 1975, s.v. tema.

5 Ibid., s.v. motivo.

${ }^{6}$ Estas definiciones se tomaron del Oxford English Dictionary, s.v. motif. 
del tema por ser una unidad casi autónoma y por su recursividad.

Una vez abstraídos estos rasgos importantes, no estamos ya muy lejos de su significación especializada. Lo único que nos sorprende es que en tematología se llama tema, también, a una historia legada por la tradición literaria, la mitología, la leyenda o el folklore: el tema de don Juan, de Fausto o de Antígona. En un primer momento, parecería contradictorio llamar tema a un concepto abstracto - 'la seducción' - y a algo tan concreto como lo es una historia más o menos cristalizada por la tradición - don Juan. El problema, sin embargo, no se resuelve en una oposición simple entre abstracto y concreto ya que, de hecho, los temas, aun cuando cubren una amplia gama que va desde la abstracción conceptual hasta la historia legada por la tradición, constituyen, en todo caso, la materia prima que ha de ser reelaborada por un autor, y por ende preexisten de manera abstracta al texto en cuestión.

Con objeto de ir precisando mejor estos conceptos, acudiremos ahora a Greimas, quien define tema como un valor, una" dissémination, le long des programmes et parcours narratifs, des valeurs déjà actualisées (c'est-à-dire en jonction avec les sujets) par la sémantique narrative"'; la tematización, por su parte, es un

procédure de conversion sémantique, la thématisation permet aussi de formuler différement, de manière toujours abstraite, une même valeur. Ainsi, par example, le valeur "liberté" peut atre thématisée. . comme "évasion spatiale" (et figurativisée à un stade ultérieur, comme embarquement pour des mers lointaines) ${ }^{7}$.

Detengámonos para reflexionar en un aspecto de esta definición: el mero concepto abstracto, valor, o categoría semántica, no deviene tema sino en conjunción con el sujeto. De tal manera que el sujeto es susceptible de resumir al tema y que por tanto, como ya lo había dicho Trousson, "qui det Prométhée pense liberté, génie, progrèss, connaissance, révolte" 8 ; piensa en lo sagrado de los lazos de sangre y de los ritos funerarios. Así, el sujeto en sus distintos niveles de figuración, ya sea como tipo o como persona-

${ }^{7}$ Cf. Greimas 1979, s.v. thème, thématisation, thématique.

8 Trousson 1965, pp. 36 ss. Es importante subrayar que Trousson concibe dos clases de tema: los temas de héroes - como el de Prometeo-que tienen alcance simbólico y tienden a una fijación menor de los elementos de la historia, y los temas de situación - como el de Antígona o los temas de personajes históricos- en los cuales se observa un mayor grado de fijación en la historia. 
je individualizado, es un centro de imantación de valores tematizados y acaba por representarlos. Precisemos, sin embargo, que el término de valor hasta aquí utilizado ha de entenderse en el sentido de categoría semántica y no de orientación ideológica. El valor estaría entonces en el nivel más abstracto de la significación. Hechas estas precisiones podemos ahora ofrecer dos nociones afines y complementarias de tema: el tema-valor, que constituye la fase más abstracta, y el tema-personaje, que sintetiza un conjunto de temas-valor en el nombre mismo del personaje. Como diría Brunel, "le nom a ici valeur d'indice".

No es entonces contradictorio hablar del tema de Fausto, de Electra o de Salomé, ya que en ellos convergen los conceptos tematizados que seleccionan y orientan el contenido posible de otros nuevos textos. Estos temas-personaje se construyen a partir de un texto original, un mito o una leyenda que luego se toman como materia prima para un nuevo texto. La tradición literaria al cristalizarlos los convierte en una especie de esquemas de orden pre-textual. No obstante, la cristalización de la historia, mito o leyenda no implica necesariamente una significación fija o predeterminada para todas sus realizaciones; por el contrario, el tema -especialmente el tema-personaje - se nos presenta como un esquema ideológicamente vacío, susceptible de proyectar los más diversos contenidos. Ahora bien, debido a que los temaspersonajes, a diferencia de los temas-valor, tienen un grado más alto de prefiguración, como la llamaría Ricourrin, una especie de "perfil narrativo" que les da su identidad como tal, es imposible "vaciarlos" totalmente de los contenidos ideológicos que marcan las versiones anteriores. De tal manera que en los temaspersonaje se observa la tensión entre los nuevos imvestimentos de orden semántico e ideológico a los que se somete un temapersonaje y la "memoria intertextual" de los anteriores.

Los temas-personaje son, entonces, una verdadera caja de resonancia intertextual. Porque, si bien es cierto que los don Juanes románticos, por ejemplo, son más afines a los Faustos y Prometeos románticos que al don Juan de Tirso de Molina, también es cierto que toda versión de don Juan declara su filiación a una tradición, a un esquema narrativo fijado por la tradición, y, por lo tanto, que toda nueva versión de un tema-personaje entabla un diálogo significante con todas las que le preceden. Y es que,

\footnotetext{
9 Brunel 1988, p. 14.
}

10 Cf. Ricoeur 1983. 
como diría Trousson, "la polyvalence du thème ne s'affirme pas seulement verticalement dans le temps, mais aussitôt horizontalement" 11 ; o en palabras de Harry Levin, un tema "is thus an avenue for a progression of ideas", pues

themes like symbols, are polysemous: that is, they can be endowed with different meanings in the face of differing situations. This is what makes an inquiry into their permutations an adventure in the history of ideas ${ }^{12}$.

Ahora bien, si el esquema de la historia de don Juan, como tal, es más o menos invariante en sus diversos avatares, se observan constantes desplazamientos en la carga temática ${ }^{13}$ - por ejemplo, de la condena en Tirso, a la salvación en los don Juanes románticos. Los desplazamientos en la carga temática son, entre otras, una forma de articulación ideológica de un material temático de naturaleza pre-textual. Los temas serían entonces literalmente pretextos ideológicos.

Un último aspecto a considerar con respecto a los temas-personaje - al igual que en los motivos - es su recursividad, porque si bien es cierto que hay una clara "asimilación' entre tema y personaje, no se concibe un tema sin estar inscrito en una tradición literaria, es decir, no se concibe un tema si no es por su recursividad. Don Juan no sería tema si no fuera por la constelación que se ha construido en torno suyo; nadie hablaría del tema de Leopold Bloom, pero sí del de Ulises. Actorialización o individualización del concepto y recursividad son elementos de definición mucho más pertinentes que la oposición abstracto vs. concreto.

Atendamos ahora a las particularidades de los motivos y a su relación con los temas-personaje. Acudiremos una vez más a Greimas para una definición más precisa: los motivos son

unités figuratives transphrastiques, constituées en blocs figés... formes narratives et/ou figuratives autonomes et mobiles, susceptibles de passer d'une culture à l'autre, de s'intégrer dans des en-

11 Trousson 1965, p. 21.

12 LeVIN 1968, pp. 140, 144.

${ }^{13}$ El concepto de 'desplazamiento de la carga temática' es de HARRY LEVIN (s.v. motif, Dictionary of the history of ideas), aunque nunca lo define con claridad; es sólo a partir de los contextos en que lo utiliza que se puede inferir su significado. 
sembles plus vastes, en perdant partiellement ou totalement leurs significations anciennes au profit d'investissements sémantiques déviantes ou noveaux [e ideológicos, habría que añadir] ${ }^{14}$.

Estas formas narrativas y/o figurativas aparecen en distintos grados de abstracción que podríamos clasificar de la siguiente manera:

a) una idea o concepto abstracto ('la rebelión'). En este sentido la confusión con el mismo concepto como tema-valor es inevitable (problemas de sinonimia que están presentes también en su sentido musical);

b) una situación de base - lo que Levin llamaría "segmento de la trama"' - que se presenta como un programa narrativo potencial ('la oposición entre el padre y el hijo');

c) espacios u objetos que se presentan como programas descriptivos potenciales ('la casa embrujada', 'el cisne', 'el laberinto');

d) imagen recurrente (palabra o frase) que puede incluso convertirse en leit-motiv; la imagen por asociación conlleva un concepto ${ }^{15}$ (cf. el "Agenbite of inwit" en el Ulises, de Joyce; el "nothing" en el Rey Lear, o el "fair is foul" de Macbeth);

e) topos: cristalización verbal de la idea (locus amoenus, 'mundo como teatro', 'la vida es sueño') $)^{16}$.

A partir de la definición que da Greimas del motivo como configuración discursiva de carácter recurrente y migratorio, resulta ya evidente el origen del conflicto terminológico que abruma a la tematología. Por una parte, tanto el tema como el motivo se caracterizan por su condición de materia prima - aunque en diversos grados de figuración - por ende en un nivel de abstracción que precede su realización en un texto concreto; por otra parte, en ambos casos se observa el fenómeno de la recursividad, la cual determina su existencia como tema o como motivo; ambos se nos presentan, en fin, como formas pre-textuales invariantes susceptibles de variaciones textuales. Para complicar aún más las cosas, al igual que los motivos, los temas tienen distintos grados de figuración, sin dejar de ser abstractos en tanto que materia pretextual, que van desde el simple concepto - o tema-valor - hasta su individualización en los temas-personaje:

a) el tema-valor de 'la seducción' que se conjuga con el tipo

${ }^{14}$ Ibid., s.v. motif, configuration.

${ }^{15}$ Levin 1968, p. 139.

${ }^{16}$ Cf. Levin, Dictionary of the history of ideas, s.v. motif, 
del 'seductor', o como diría Greimas, el recorrido temático 'pescar' que se resume en el rol actancial 'pescador';

los temas-personaje con diversos grados de figuración:

b) los temas de héroe, más libres, en los que se resumen diversos valores o conceptos, tales como el tema de Prometeo;

c) los temas de situación, que tienen una mayor fijación, individualizan situaciones de base o programas narrativos potenciales, tales como el tema de Antígona;

d) los temas de figuras históricas que alcanzan un máximo grado de fijación e individualización por estar ligados a una época y a un lugar concretos, tales como el tema de Napoleón o el de Thomas a Beckett.

Es evidente que tanto los temas como los motivos aparecen en distintos grados de figuración, sin dejar de ser abstractos. No es, insistamos, en la oposición abstracto vs. concreto que reside la diferencia, sino en otro nivel. Hemos dicho que el tema se asimila en el personaje; aunque de hecho casi siempre se trata de varios valores o categorías semánticas relacionados entre sí y que entran en conjunción con el sujeto. Es en esa pluralidad de valores que reside la polivalencia de un tema-personaje: bajo la cobertura de un nombre propio, un tema-personaje es una sintesis de diversos motivos y de temas-valor, ordenados e interrelacionados de tal manera que dibujen un perfil narrativo que le confiera identidad al tema. Recordemos simplemente la observación de Trousson: "qui dit Prométhée pense liberté, génie, progrès, connaissance, révolte..." Cada una de las actualizaciones del tema-personaje puede hacer hincapié en uno u otro valor; es decir, uno de los temas-valor tendrá un peso mayor; será en él que descanse la carga temática y por lo tanto la orientación ideológica de la obra.

Estas consideraciones nos permiten ahora precisar el concepto de Harry Levin de 'desplazamiento de carga temática', pues si en un tema-personaje coexisten en distintos grados de actualización y de jerarquización varios conceptos o temas-valor, es evidente que ciertas reelaboraciones pondrán el énfasis en uno más que en otro. Es por ello que en las diversas realizaciones de un tema-personaje se observan desplazamientos en la carga temática; estos desplazamientos de un tema-valor a otro tienen, en sí, un fuerte valor ideológico. El don Juan de Tirso se condena, el de Zorrilla se salva; en el solo desplazamiento de carga temática queda implícita toda una postura ideológica.

Ahora bien, si el tema-personaje es una unidad compleja, resultado de una combinatoria, el motivo en cambio se presenta 
como unidad simple ya sea en forma de microrrelato o de figura. La situación de base a la que se refiere Trousson - tal como la oposición entre padre e hijo- no es otra cosa que un programa narrativo potencial. Así un tema-personaje puede considerarse también como la combinación de varios motivos, ya que en su sentido más abstracto hay un punto de coincidencia en la significación de tema-valor y de motivo. La combinación es más o menos estable, más o menos compleja - el mínimo común denominador del tema estaría determinado por la presencia de aquellos motivos que son absolutamente esenciales para mantener su identidad. Los motivos, en tanto que unidades simples, tienen una mayor libertad de inserción y una mayor capacidad de migración. El motivo de 'la cita en la tumba', por ejemplo, en tanto que programa narrativo potencial, es esencial para la identidad del tema de Píramo y Tisbe, pero como unidad transfrástica más o menos fija mantiene su autonomía al inscribirse en diferentes contextos que nada tienen que ver con ese tema, sin que por ello pierda su identidad como motivo. Está presente en el Romeo y Julieta de Shakespeare, sin que por ello esa obra sea una de las actualizaciones del tema de Píramo y Tisbe, y sin que por ello el motivo de 'la cita en la tumba' pierda su identidad como tal.

\section{Métodos de trabajo}

Tradicionalmente la tematología ha investigado la evolución de los temas con un enfoque esencialmene histórico, de lo que han resultado trabajos de compilación o de inventario. No en vano el libro-biblia de la tematología se llama Stoffgeschichte, historia de los temas. La otra vertiente importante del estudio de temas y motivos, la que quizá está más cerca de la etnoliteratura, es la taxonómica; no hay sino recordar las 2000 situaciones dramáticas, de Souriau, o el abrumador Motif-index of folk-literature, de Stith Thompson que enumera y clasifica 40,000 incidentes sacados de cuentos, mitos, fábulas, libros de aventuras, baladas, libros de chistes, los muy didácticos exempla, y toda clase de textos narrativos. Desde esta perspectiva metodológica, los estudiosos se han abocado a clasificar la materia prima de la literatura, o se han orientado hacia la historia literaria y la historia de las ideas; su meta: demostrar la continuidad de la tradición histórica y literaria de un tema. Esta última es claramente la aspiración de Trousson, quien descarta la vía taxonómica, pues 
respecter l'ordre chronologique, entasser des documents, accumuler des dates et des noms, c'est le rôle d'un dictionnaire; tracer des pistes dans la jungle des interprétations et des transformations d'un thème dans le cadre de l'histoire des idées, c'est l'affaire de la thèmatologie ${ }^{17}$.

Pero "la literatura - alguien ha dicho- es una incesante redistribuidora de mitos" y, tal vez, hasta de métodos. Hoy en día, incluso los trabajos de clasificación parecen estar imbuidos de una conciencia textual, como bien lo testifica el extraordinario trabajo de clasificación crítica bajo la dirección de Pierre Brunel, Dictionnaire des mythes littéraires (1988). A diferencia del abigarrado Motif-index de Thompson que intenta engullir el universo narrativo entero y devolvérnoslo en fragmentos, este diccionario parte del presupuesto de que el mito en sí se distingue con dificultad del tema debido a que el "mito está animado por un dinamismo que es de orden narrativo" (p. 8). Su modo de transmisión y su consignación por medio de la escritura lo convierten en un hecho literario. Más aún, como lo afirma Dumézil "nous ne connaissons les mythes que par la littérature. . c'est à partir de textes ou de traditions littéraires qu'on avance des hypothèses sur ce qui les a précédés"'18.

Así pues, por su modo de transmisión narrativo, así como por su modo de inscripción que es esencialmente textual, el mito es en sí un fenómeno literario o textual. Algunos mitos son incluso doblemente literarios, como es el caso del de Fausto, Tristán e Isolda, o don Juan. Finalmente, según Brunel, hay una nueva categoría, constituida por aquellas figuras, eventos históricos, o incluso lugares, que la literatura misma ha transformado en mitos literarios. Así, y cada vez de manera más evidente, el estudio de temas y motivos queda inscrito en una teoría de la textualidad que puede darse en la modalidad del mitoanálisis o en la de un análisis intertextual capaz de identificar al tema "mediante la memoria de figuraciones anteriores", como diría Guillén.

Los grados de presencia de un texto en otro, así como los grados de textualidad que puedan darse en esta relación son tan variados que justifican a Gérard Genette en su propuesta de una teoría de la transtextualidad. Para él, la transtextualidad, o trascendencia del texto, es "tout ce qui met [el texto] en relation mani-

17 Trousson 1965 , pp. 30-31

18 Apud Brunel 1988, p. 11. 
fieste ou secrète, avec d'autres textes'"19. Son cinco las diferentes formas de transtextualidad, en las que se observan grados crecientes de abstracción e implicación:

1) La intertextualidad, definida como una "relación de copresencia entre dos o más textos". Las relaciones intertextuales, como las transtextuales en general, se pueden dar en distimtos grados de abstracción: desde el carácter puntual de la cita, hasta las formas más sutiles e indirectas de la alusión. Es claro que esta forma de relación es la dominante en las constelaciones temáticas que hemos estado estudiando: desde la referencia directa y explícita al texto anterior, hasta la alusión indirecta por medio de situaciones semejantes o nombres que aluden a los de los personajes originales, como en el caso de la obra de O'Neill, Mourning becomes Electra.

Ahora bien, si en lo general la relación intertextual se define en términos de dirección e indirección, al interior de estos dos modos se observa todo un espectro que va de lo concreto a lo abstracto. En la forma directa de relación intertextual, el grado de presencia más concreto es la cita puntual, con referencias precisas al texto del que proviene; un grado más abstracto se observaría en la paráfrasis y toda forma de asimilación del texto citado al texto "anfitrión".

En la forma indirecta de la relación intertextual, la alusión, también se observan diferentes grados de indirección:

a) alusión textual, en la que un lexema o grupo de lexemas proveniente de otro texto se asimila al nuevo contexto. Esta forma de alusión está muy cercana a la cita; la única diferencia que las marca es la indirección: la cita declara su origen, la alusión textual lo calla;

b) alusión tópica: referencia más o menos velada a eventos recientes, y por tanto a textos históricos, periodísticos, etcétera;

c) alusión personal: referencia a eventos de la vida del autor, mismos que con frecuencia acaban constituyendo un "texto" de naturaleza autobiográfica, como en el caso de un Yeats quien escribió no una sino varias Autobiographies;

d) alusión metafórica, en la que el elemento aludido del hipotexto se utiliza como el vehículo, o grado manifiesto, del nuevo tenor poético, o grados construidos de la significación metafórica en el hipertexto (cf. la alusión a la "Última Cena" para expresar la relación entre Macbeth y Duncan, I, vii, 1-28);

${ }^{19}$ Genette 1982, p. 7. 
e) alusión estructural, en la que un texto sugiere o utiliza para su construcción la estructura de otro (característica predominantemente hipertextual). Ejemplo de esto sería la estructura musical en los Four quartets de T. S. Eliot, o la estructura de la Eneida, réplica de la de la Odisea ${ }^{20}$.

2) La paratextualidad, definida como una relación

généralement moins explicite et plus distante, que... le text proprement dit entretient avec ce que l'on ne peut guère nommer que son paratexte: titre, sous-titre, intertitres, préfaces, postfaces, avertissements, avant-propos, etc.; notes marginales, infrapaginales... épigraphes; illustrations... et bien d'autres types de signaux accessoires... qui procurent au texte un entourage (variable) et parfois un commentaire, afficiel ou officieux, dont le lecteur le plus puriste et le moins porté à l'érudition externe ne peut pas toujours disposer aussi facilement qu'il le voudrait et le pretend ${ }^{21}$.

En el caso del Ulises, de Joyce, por ejemplo, es inconmensurable el valor significante de la relación paratextual, ya que es el título mismo el que orienta una lectura que lo ponga en relación hipertextual con la Odisea. Y ni qué hablar del guiño intertextual y paratextual en aquella obra de Girodoux que incluso nos ahorra la investigación relativa a cuántos Anfitriones se han escrito antes del suyo.

3) La metatextualidad es la relación crítica por excelencia que une a un texto con otro del que habla, sin que la cita sea indispensable. Después de todo, ya lo apuntaba Guillén, es el crítico quien "sin cesar elige, extrae, cita"; es por ello que "las formas y los temas, más que entidades discretas, son elementos parciales cuyo montaje se debe en definitiva a la intervención del lector" o el crítico. Es él, en gran medida el responsable de la constitución de un tema por su afán de reagrupar los textos desde una perspectiva temática.

4) La hipertextualidad describe una relación de derivación de un texto, o hipertexto, con respecto a otro anterior, o hipotexto. El tipo de derivación en esta relación no es, sin embargo, una relación de comentario crítico - como en la metatextual- sino una operación de transformación. El hipertexto no puede existir cabalmente sin el hipotexto, pero no sólo habla de él o lo cita, sino que lo transforma para construir un nuevo texto con propiedades lite-

${ }^{20}$ Cf. Preminger 1976, s. $v$. allusion.

21 Genetre 1982, p. 9. 
rarias. Así, la Eneida y el Ulises son ambos hipertextos de un mismo hipotexto: la Odisea.

Es importante subrayar que las relaciones hipertextuales engloban y trascienden las intertextuales. Es claro que un hipertexto tiende a relacionarse con el hipotexto si no mediante la cita directa por lo menos por medio de constantes alusiones a él. La diferencia reside en que, en la relación hipertextual, las relaciones intertextuales entre ambos textos son sistemáticas y se extienden a la totalidad del texto. Además, la relación hipertextual está basada en la estructura global propuesta por el hipotexto, que el hipertexto replica en mayor o menor grado. Este carácter sistemático y totalizador de la relación hipertextual le da una cierta autonomía y, al mismo tiempo, le permite incorporar otro tipo de relaciones transtextuales -intertextuales y paratextuales en especial. La alusión, por ejemplo, característica de las relaciones intertextuales, también lo es de las hipertextuales, con los mismos modos y grados de presencia. Asimismo, como lo hemos visto en el caso del Ulises de Joyce o del Mourning becomes Electra de O'Neill, con frecuencia la relación hipertextual se funda en una relación paratextual; es el título, el subtítulo de un texto, a veces incluso un epígrafe, lo que revela una relación hipertextual implícita que de otro modo quizá pudiera pasar desapercibida.

5) La architextualidad es la relación más abstracta e implícita que se presenta como una relación de filiación genérica. "Como es bien sabido la percepción genérica orienta y determina, en gran medida, el «horizonte de expectativas» del lector y, por tanto, la recepción de la obra'. La relación architextual también define, además de la filiación genérica, la conciencia de formas y convenciones dentro de la tradición literaria. De hecho un architexto es una suerte de síntesis abstracta de un sinnúmero de textos semejantes que configura una especie de texto ideal al que el texto en cuestión ha de conformarse o contra el que ha de rebelarse. La fijación de un tema, su pertenencia a una tradición, lo hemos dicho, se debe a la recursividad; sin ella no se concibe el tema. Ahora bien, el concepto mismo de tema, su unidad, reside en un mínimo común denominador de todas sus actualizaciones textuales. Girodoux sabe muy bien que al nombrar su obra la pone en relación manifiesta y concreta con las 37 que la han precedido pero también con ese Anfitrión-tema que no es sino el architexto que ha surgido de las 37 anteriores.

Hasta hoy todo el peso de la investigación en el área de los temas y los motivos ha recaído en la clasificación, en el rastreo 
de la evolución de los temas y motivos a lo largo de la historia literaria y su relación con la historia de las ideas; lo que se ha descuidado es un análisis más fino que dé cuenta de las transformaciones textuales y la manera en que se relacionan con otros textos. Una teoría de la transtextualidad nos permite explorar el fenómeno del tema que se constituye en tradición, en las expectativas genéricas que construyen ciertos temas y desafían otros; qué es lo que ocurre al operarse una transmodalización entre el hipertexto y el hipotexto; qué formas de transvaloración permiten describir las articulaciones ideológicas de un texto dado; qué significación y qué grado de presencia tiene un texto en el otro, etcétera.

Examinemos brevemente tres textos que actualizan el tema de Salomé: "Hérodias" de Flaubert, "Salomé" de Laforgue, y Salomé de Oscar Wilde. Flaubert se basa en los Evangelios de Marcos y Mateo -a su vez basados en hipotextos concretos y tradiciones anteriores- y en las Antigüedades judaicas, de Flavio Josefo, con el propósito, entre otros, de hacer una reconstrucción histórica y política de la época. Laforgue toma el cuento de Flaubert como hipotexto básico y lo parodia. Un análisis minucioso de todas las operaciones de intertextualidad a las que recurre Laforgue - la paráfrasis y todo tipo de alusión- podría revelar el poder corrosivo y subversivo de esta parodia, que arrasa desde el contenido ideológico de "Hérodias" hasta las técnicas descriptivas de Flaubert. Desplaza además el centro de atención de Herodias a Salomé, desvalorizando además, y de modo paródico, la carga temática sobre lo político, característica del hipotexto, al sustituir la danza de los siete velos por un interminable, e indescifrable, discurso sobre el inconsciente que pronuncia Salomé frente a los invitados.

Ahora bien, el cambio del centro de atención de la madre a la hija es tan fuerte hacia finales del siglo xIX que ya no es Herodias sino Salomé quien acaba resumiendo el tema. En la obra de Oscar Wilde, cuyo hipotexto es también el relato de Flaubert, se opera un desplazamiento de carga temática, de lo político a lo erótico; además se observa una serie de transvalorizaciones que le dan a esta obra su peculiar carácter de perversión, entre otras una transvalorización de la decapitación, no ya como venganza sino como posesión sexual. Finalmente, un estudio detallado de la transmodalización en esta obra con respecto al hipotexto - el paso del modo narrativo al modo dramático- podría ser muy ilustrativo de los problemas y fallas que tiene la obra de Wilde. 
Concluyamos observando que si bien es importante la función taxonómica en los estudios de temas y motivos, pues es la que nos permite el acceso a diccionarios y a toda suerte de compilaciones; que si es de igual importancia, como afirma Trousson, "rastrear pistas en la jungla de las interpretaciones y de las transformaciones de un tema en el marco de la historia de las ideas"'22, es de suma importancia también poder estudiar los temas desde la perspectiva de las múltiples y complejas relaciones que establece un texto con otros, pues esto permite describir con mayor acuciosidad las formas de articulación ideológica de un texto concreto.

Ha sido mi intención a lo largo de este trabajo abordar dos problemas interrelacionados. En un primer momento, una exploración a nivel conceptual de los términos que tradicionalmente ha utilizado la tematología nos ha permitido alcanzar una precisión terminológica mayor, aun cuando paralelamente hayamos llegado a un nivel de mayor complejidad. Un segundo aspecto estudiado ha sido el problema de los métodos de trabajo: es de suma importancia hacer patente que el hecho de clasificar temas y motivos, o de rastrearlos al través de la historia literaria y de las ideas, no son ni las únicas tareas posibles, ni las únicas valiosas en este campo de estudio; un análisis textual e intertextual es igualmente útil. Porque si bien es cierto que como materia prima los temas y motivos -independientemente de su grado de figuración- tienen una existencia pre-textual, es igualmente indudable que su existencia pre-textual es, a un tiempo, plenamente textual, que incluso a nivel de mitos, leyendas y tradiciones hay una consignación por escrito en innumerables textos y que al operar una síntesis que nos permita una formulación continua y coherente del mito o la leyenda no estamos haciendo otra cosa que construir un architexto. Sin hablar más de aquellos mitos literarios que lo son doblemente por haber surgido de textos literarios concretos y que un análisis hipertextual puede describir de manera más eficiente. Y es que hoy en día, en los estudios literarios comparatistas, una teoría de la textualidad y de la transtextualidad conduce a un estudio verdaderamente interrelacionado de la literatura. Sin una teoría general del funcionamiento textual e intertextual, todos los estudios de imitación, influencias, convenciones y formas literarias, temas y motivos se mantienen en un 
aislamiento propiciado por enfoques que aún proclaman su filiación positivista.

Luz Aurora Pimentel Universidad Nacional Autónoma de México

\section{BIBLIOGRAFÍA}

Brunel, Pierre (ed.) 1988. Dictionnaire des mythes littéraires. Éds. du Rocher, Paris.

Genette, Gérard 1982. Palimpsestes. Seuil, Paris.

Greimas, A. J., \& J. Courtès 1979. Sémiotique. Dictionnaire raisonné de la théorie du langage. Hachette, Paris.

Guillén, Claudio 1985. Entre lo unory lo diverso. Introducción a la literatura comparada. Crítica, Barcelona.

Levin, H. 1968. "Thematics and criticism", en P. Demetz et al. (eds.), The disciplines of criticism. Yale University Press, New Haven-London. (Una versión ligeramente modificada apareció en su libro Grounds for comparisson, Harvard University Press, Cambridge, MA, 1972.)

Moliner, María 1975. Diccionario de uso del español. Gredos, Madrid.

Preminger, Alexander (ed.) 1976. Princeton Dictionary of poetry and poetics. Princeton University Press, Princeton, NJ.

Ricoeur, Paul 1983. Temps et récit I. Seuil, Paris.

Trousson, Raymond 1965. Un problème de littérature comparée: les études de thèmes. Essai de méthodologie. Lettres Modernes, Paris.

Wellek, René, \& Austin Warren 1949. Theory of literature. Harcourt, Brace \& World, New York. 
